\title{
Selection of an Appropriate Thresholding Technique to Quantitate Fluorescence
}

\author{
Jeffrey C. Hanson* \\ * Eli Lilly and Company, Lilly Corporate Center, Indianapolis, IN 46285
}

Keywords: Quantitation, Fluorescence

Fluorescence is often used to generate qualitative measures. Because of the nature of fluorescence (e.g., non-specific fluorescence in tissue, quenching), generating any form of quantitative measures requires careful sample preparation, controlled acquisition parameters and a consistent set of guidelines for thresholding and counting. Evaluating different guidelines for thresholding is the subject of this paper.

The baseline quantitative measurement for fluorescence is a measure of total area of fluorescence in the area of interest. Small changes in this threshold can dramatically affect how much area is counted as fluorescent. The different techniques choosing a threshold level compared here are:

- single constant "best" threshold across all images

- best threshold for each image based on user perception

- threshold based on histogram of each image

- threshold based on histogram of area of interest

Each of these techniques can be rated based on the kinds of measurement error that they could introduce, the amount of time required for processing, and their ability to respond to varying fluorescent conditions. The constant threshold takes the least time, but could generate larger errors when background fluorescence levels vary. The best user-perceived threshold produces results most closely matched to user perception, but introduces more human bias and takes longer. Both of the histogram-based techniques are quick and reduce human bias, but introduce another kind of error in images where the signal covers a significant portion of the image.

\section{REFERENCES:}

[1] J. C. Russ (2002), Image Processing Handbook, Fourth Edition, CRC Press, Boca Rotan, FL.

[2] A. Kriete (1992), Visualization in Biomedical Microscopies, 3-D Imaging and Computer Applications, VCH Publishers, New York, NY.

[3] J. B. Pawley (1995), Handbook of Biological Confocal Microscopy, Second Edition, Plenum Press, New York, NY. 


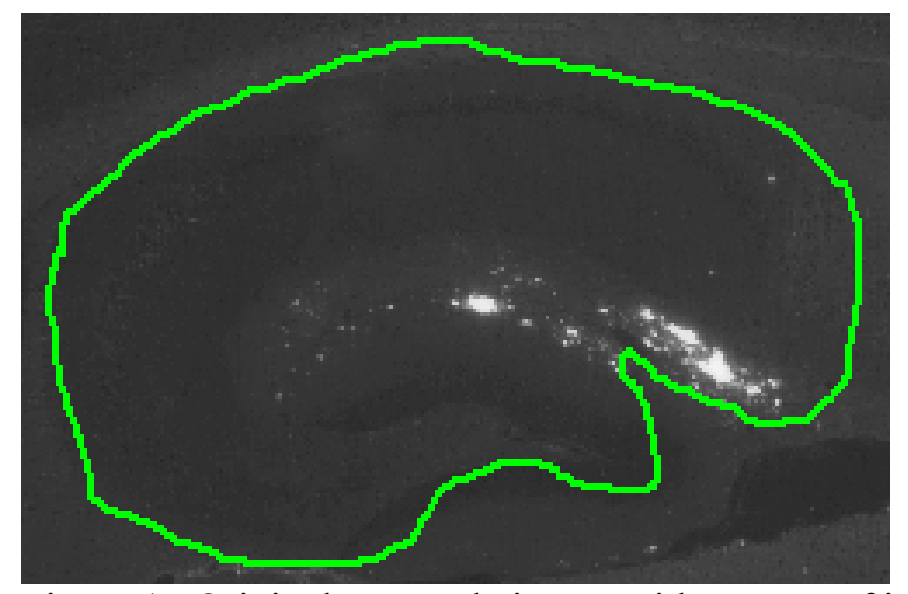

Figure 1. Original grayscale image with an area of interest (AOI) outlined in preparation for quantification.

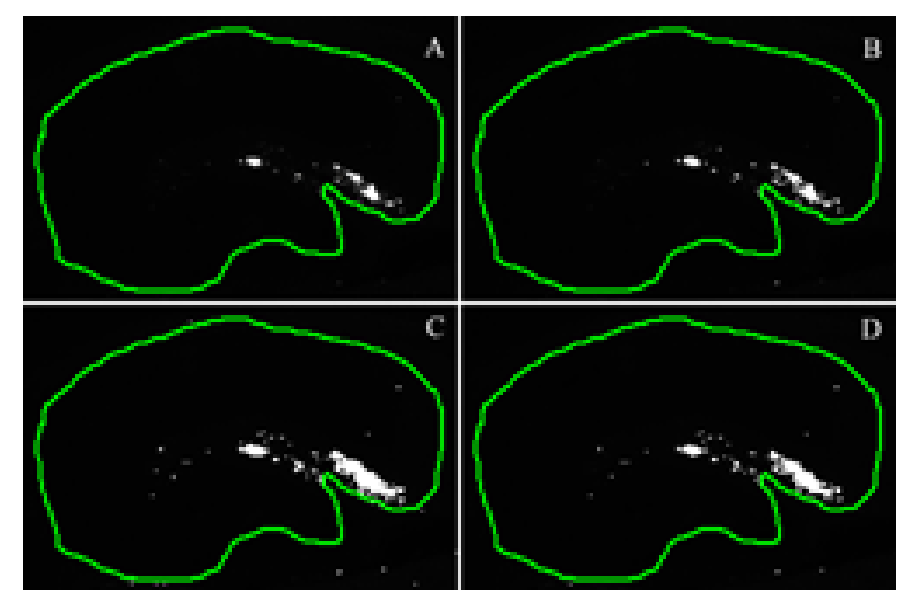

Figure 2. Image thresholded using various thresholding techniques, 107 pixels from a constant threshold (A), 207 pixels from the "best visual" threshold (B), 563 pixels from a histogram-based approach (C), and 422 pixels from a histogram of AOI-based approach (D). Note that the different approaches can lead to widely different results. 